\title{
An OLAP Service for Data layer of Collaborative Wireless Sensor Network Stack
}

\author{
Kamlendu Kumar Pandey, Veena Jokhakar
}

\begin{abstract}
The sensor networks generate immense data and often flood the network traffic with it communication to the cluster nodes and gateways. The enormous data generated has to be analyzed in a uniform manner to extract maximum benefit from the data. The problem is all the sensor nets are on diverse platforms and have different data structure. Effort has been done in this paper to have a uniform sensor data structure and a registry to discover the sensors. A model is proposed with a complete stack of services for the sensor networks. The data service layer is implemented in a practical way with an experimental set up for data gathering and then its analysis by prevalent OLAP techniques with training and validation on trial basis
\end{abstract}

Keywords: Wireless Sensor Networks, Sensor Registry, OLAP, Data Analytics

\section{INTRODUCTION}

The upcoming technologies like High Speed Computing, Artificial Intelligence and Big Data Analytics are finding their applications in diverse areas which generate Sensor Networks and its application like weather monitoring, defense surveillance, home automation etc are the fit candidates for such applications. Database technologies are opening up to have a noteworthy impact in the promising area of wireless sensor networks (sensor nets). Sensors are used in various fields like in manufacturing industries to measure various parameters like temperature, pressure, speed, etc. as well in weather forecasting to measure humidity, weather conditions at various locations. In recent times Data Analytics has proved to be more useful to replace conventional methods of data analysis and decision-making. BI is basically a blend that consists of various technologies like Data warehouse with all its components wherein the data is extracted, integrated, cleansed, transformed The upcoming technologies like High Speed Computing, Artificial Intelligence and Big Data Analytics are finding their applications in diverse areas which generate enormous amount of streaming data in real time. Wireless Sensor Networks and its application like weather monitoring, defense surveillance, home automation etc are the fit candidates for such applications. Database technologies are opening up to have a noteworthy impact in the promising area of wireless sensor networks (sensor nets).

Revised Manuscript Received on May 30, 2020.

* Correspondence Author

Kamlendu Kumar Pandey, Department of ICT, Veer Narmad South Gujarat University, Surat, India, Email: kspandey@vnsgu.ac.in

Veena Jokhakar*, Department of ICT, Veer Narmad South Gujarat University, Surat, India, Email: vajokhakar@vnsgu.ac.in

(C) The Authors. Published by Blue Eyes Intelligence Engineering and Sciences Publication (BEIESP). This is an open access article under the CC BY-NC-ND license (http://creativecommons.org/licenses/by-nc-nd/4.0/) enormous amount of streaming data in real time. Wireless

Sensors are used in various fields like in manufacturing industries to measure various parameters like temperature, pressure, speed, etc. as well in weather forecasting to measure humidity, weather conditions at various locations. These data are collectively collected is enormous worldwide.

BI is basically a blend that consists of various technologies like Data warehouse with all its components wherein the data is extracted, integrated, cleansed, transformed and then loaded in to a relational multidimensional database, Information and knowledge representation technologies like data mining, OLAP (Online Analytical Processing), Statistical analysis, Executive information systems, decision support system etc.

Organizations may use different BI tools and technology to develop different types of business intelligence applications. Some of the features that BI tools support are typically data analysis. This part of data analysis may be performed at both real time and historical data.

The various handling of data done over the historical data is performed usually on a data warehouse and later to perform analysis on both historical as well as real time we work on various forms of OLAP and alert messaging or notification services.

However it is vital that the tool should be easy to realize, control and flexible enough to have a room for prospected changes and complexities of business. We hence propose a hybrid model of Sensor net and OLAP.

The use of Wireless Sensor Networks is now becoming more rampant in various problem areas from environmental monitoring to surveillance and vehicle tracking. This technology is now coming out of labs to actual implementation in the fields. Achieving speed and quantity is the biggest confront on the data sensed and disseminated in the network. All the data actually is directed towards the sink node. If the data is not handled in right way in the sink node, the data is lost.

Hence there has to be a way out to carry out the mechanism of packets to sink node is carried out perfectly. Based on the packet structure of the sensor, the data should be read. Middleware helps to a great extent at this point.

An Arrangement of a server that can be configured undefined number of times based on the sensing device can be used to deal with the data, storage of data in the way it needs to be. The storage can be range from XML file to sophisticated RDMS. After being stored, the data has to be utilized to decision making, trend analysis of the data captured.

This paper uses GSN a famous middleware developed by Ali Sulehi, describes all the steps from installation of middleware, custom code writing for storage of data. Finally the storage data is represented in instantaneous graphs with warning system.

\section{Published By:}


II.

\section{THE CURRENT RESEARCH}

The data sensed by the sensors finally comes at the sink node and is aggregated by application present on the host machine. This application acts as middleware between sensors and the datastore. Numerous works have been done in this context. Al-jaroodi [2] has given the detailed list and summary of all the work of middleware softwares which with their specialities and limitations do the task of data collection and storage. SstreamWare [2] is also a mediator for sensor data. It gives a query based service to acces data from sensors deployed on diverse platforms. The main challenges handled I this work was the way to deal with the management of diverse sensor platforms and to execute complex queries. The scaling of the clusters and data containers is also discussed. The University of Melbourne , alsoin their Cloud computing and distributed System labs have developed a National Open Sensor Web Architecture. SW 2.0[3] developed by active collaboration of Open Geo Spacial Consortium is also talking about the streaming data from sensors.

Open Access Sensor Information System [1] is based on publish-subscribe technolgy of web services based on a well defined network stack and Acive Objects System. The application is based on layered architecture where main functionalities of sensors form the core broker which is involved in finding services, fallback procedures, health check, orchestration, security and dynamic configuration

Ali Sulehi et al developed GSN [6] called Global Sensor Networks which can be regarded as one of the best open source and simple solution to capture the streaming data from Sensor Networks. He has arranged the application layers in Wrapper Classes and Virtual Sensors . The user has a freedom to choose and configure its own database and improve upon the existing application. This has a readymade application dealing with various sensors like , camera, file system, micaz boards base on ZIGBEE etc. Botts et al [7] is rlying on the integration of Sensor data with OGC while Kobaika et al [8] also tries to have an sensor architecture based on different platforms and architectures. The GSN developed by Ali Sulehi is open to adding any new Sensor Boards provided you have a compatable driver and library pertaing to the packet structure of the underlying sensor boards. The developers can try sensor boards like plain arduino or TinyOS based micaz data acces boards [9]. The front end is a Web UI which helps to see and change the configuration and observe the live data

\section{IDENTIDICATION OF PROBLEM}

The deployments of sensor networks is mostly private and organisational in nature. You wont find a public sensor networks except in State Meteriological Stations. The data being streamed from all private networks is used in a small domain and for a specific purpose. If all this data can be shared across globe to facillitatae researchers, decision makers and policy designers. The main bottleneck is we don have standard to set up such data sharing and querrying standards right now.

We need to focus on following aspects

1. Design a collaborative Stack to facillitate the activities from data acquisition to data visualizations

2. Go for standard data analysis techniques from OLAP, Warehousing and Data mining

In this paper we peek into the aforesaid issues.
IV.

\section{BACKGROUND OF COLLABORATIVE} APPROACH

To say that just having the cloud based approach is going to solve the problems of collaborative computing in sensor networks is not true. The problem require an overhaul of the entire architecture which has been followed till now. We started this overhauling in three phases

1) We have proposed a Web DNS like registration system for all king of sensor networks and call it a Sensor Registry Services (SRS)

2) We proposed an complete stack of Services to be used in the process of sharing the sensor networks data.

3) We propose creating an universal Collaborative data store and the ways to analyse the streaming data using modern data analytics techniques

The Uniform Registration Process

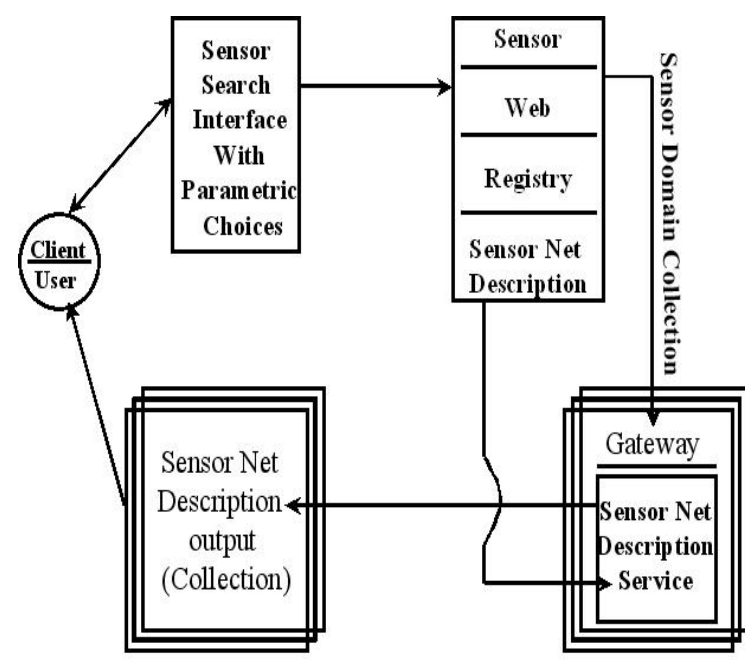

Gateway with httpd, JBI server \& BPEL repository

Fig. 1 Sensor Web Registry

Looking to the identidication of problem in section III a registry was proposed by me and Dr. S.V. Patel [11,12] in which emphasized the requirement of such a framework which can be universal and can be agreed upon technically, legally and politically just in the line with web DNS. The current cloud computing platforms have made this idea really feasible. The technology has changed in the mean time and there are some modifications in the proposed registry. Previously the registry used to register all WSNs as service based on SOAP - WSDL standsards but now this standard is revised to REST Pull-Push Standards with micro services and container managed Devops.

The readers of this paper may kindly refer the [5] and [6] for more ellaborate information. In short all the configurations from Owner registration, network registration, Data category, field to be shared and mapping throgh a uniform data format, location etc are done. This also defines all authentication and role based access. The idea is a normal user can vie the data of data of intrest filtered by location, region, limits, trends etc. The subscribers to thsis service may have access limited to their autherity and prebailing legal system in their rgion. This approach can make this world more more responsive to any threat or outcome like pandemics or natural disasters.

Published By: 


\section{THE COLLABORATIVE WSN SETUP}

In continuation with uniform Sensor Registry, it was decided to go for a proposal for a complete mature stack of cloud based services which can be accessed by the subscribers of SRS. The scaffolding of SRS has to be used to build a complete structure of stadards and their implementation for various services. This stack was proposed in [13] but is refined now with keeping an emphasis on Data Layer. The earlier services stack is already shown in fig. 2. Only Data layer is emphasized in this paper. The reader may read the paper for elaborate information on Entire Service Stack

For the sake of connectivity we woulldVir like to name these layers and services as follows:

1. Network Layer

(a) Container Virualization Services

(b) Routing Services

(c) VM Services

(d) Storage Services

(e) Load balancing Services

2. Administration layer

(a) Collaborative SNregistration Services

(b) User/Subscriber Registration Services

(c) Access and Authorization Services

(d) Pricing and Metering Services

(e) Mediation Services

3. Repository Layer have a central data store of

(a) Registered WSN Records

(b) Registered Subscriber Records

(c) Authorization and public Key Records (d) micro services registration records

(e) cloud records

4. Data layer

The services in this layer the services are as under

(a) Data Aquisition Service

(b) Data Cleaning and Disseminition Service

(c) Pull and Push Services

(d) Warehouseing Service

(e) Mining Service

(f) Event Notification Servicer

(g) Mapping Services

(h) Monitoring Services

(i) Data Visualization Service

All the layers are shown in fig.2 as collaborative stack service in Wireless sensor Networks

The emphasis of this paper is the visualization of streaming dataof WSN in the data layer most of the above layers will be used from the stack. The service here is realized by a real time experiment done with MicaZ motes for weather data aquissition. The Micaz motes are based on tinyOS standards and network packets transmitted with AODV routing protocol is received at the gateway node connected to host system by a USB device. The experimental setup is described in Fig 3. We have adopted the application of GSN by Ali-Sulehi and configuration and customizations were done to the software to suite the local requirements abd devices. . It was planned to collect the data by a Pull Service of Data Layer and subject it to Aquisition service. The backend datastore used in this case is MySQL and Postgre sql.

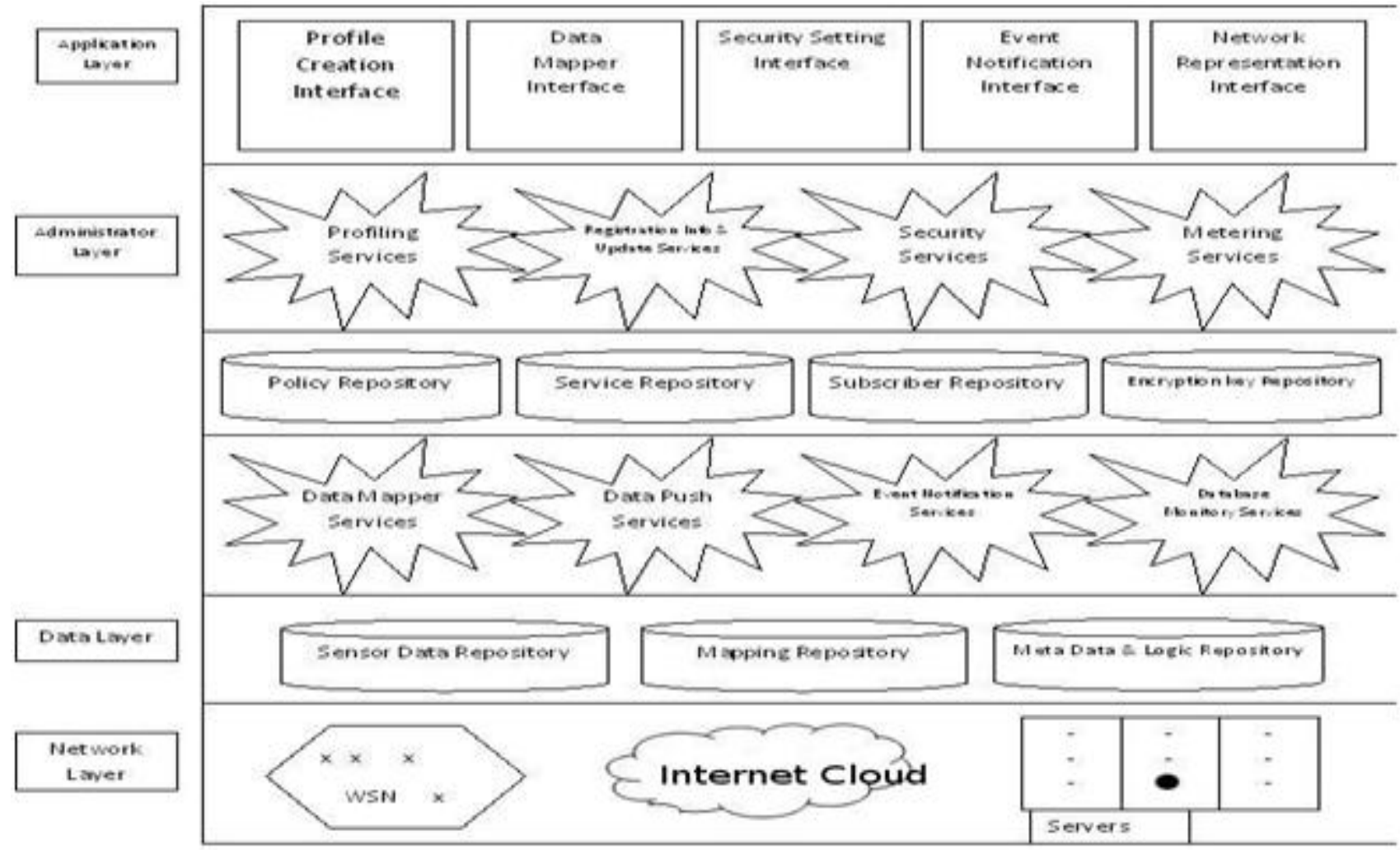

SERVICE STACK FOR COLLABORATIVE WSN

Fig. 2 A collaborative WSN stack

Published By:

Blue Eyes Intelligence Engineering
\& Sciences Publication 


\section{THE EXPERIMENTAL SETUP}

To capture the data in the proposed service stack following experimental setup was done
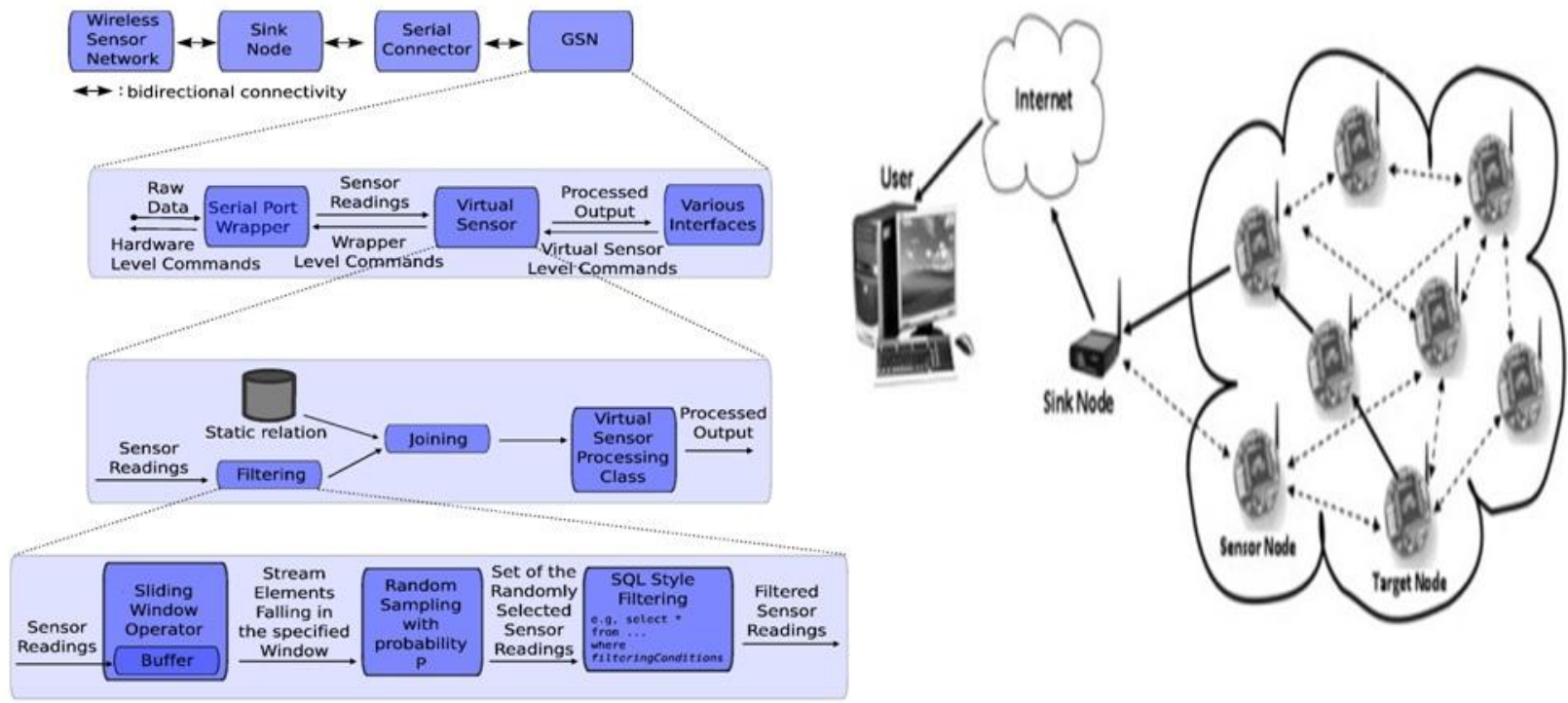

Fig. 3 The sensor placement and Data capturing setup with Global Sensor Net (Courtesy : www.redcad.org, Dr. Ali Sulehi )

The experimental setup is having the following configuration

1. Micaz Cross bow motes : 5 Nos.

2. Micaz Gateway Board 1 No.

3. Host Machine :

OS : Ubuntu 18.03

Middleware : GSN on host

4. Database : MySQL / Postgre SQL/ XML/ CSV

5. Programming Language : Java / Java EE

6. Data Visualization tools : Python Libraries with Machine learning capabilities, ETL for data and query view dissemination.

7. AWS S3 instance for data storage to be accessed by OLAP services.

8. Web Interface

Data aquissisoned :

(a) Temperature

(b) Humidity

(c) Air Pressure

(d) Light Intensity

(e) Horizonal Acceleration

The data undergoes all the operation mentioned above in various layers. The role of virtual sensors , data mappers, visualize it more meaningfully.

\section{DATA AND INFORMATION ANALYSIS AND VISUALIZATION}

As huge data is being generated in every frild today, and there is no hardware that possess the capacity to store such huge capacity of data, the data in recent years have been started to be stored in cloud that holds the data that are collected,monitored,stored,dissiminated and process the various incoming data from various WSN.It deals with the query produced by subscribers and deliver the data after (f) Vertical Acceleration XML artifacts are considerd to design an OLAP model to

optimizing and executing it. There are various services that take care of the whole process that can be listed as DPPS (Data Pull and push service), DMS (Database Mapping Service), DCDC (Data Cleaning and Dissemination Service), DWS (Data Warehousing Service), DMS (Data Mining Service), ENS (Event Notification Service), DVS (Database Virtualization Service) and DMS(Database Monitoring Service).

The various depositories that will be needed for the proper functioning of the services are as follows:

i)The Real Time Data Repository - that stores the incoming data from various WSNs

ii)Meta Data and Logic Repository - that specified the metadata function, triggers, structures, procedures, function, triggers etc.

iii)Mapping Repository : That hold the data mapping details for every WSN with Cloud's transactional database

iv)Historical and Summarized Data Repository : that deals with the data warehouse and is maintained by cloud.

\section{A. DPPS:}

Based on the agreements agreed by the WSNs the cloud setup pulls the dataset from WSN organization and in the similar fashion the current cloud can also be a data provider for other cloud, then the data push services are needed.

\section{B. DMS:}

The data is sensed from various WSNs, which individually have their own data definition and dictionary. Hence a common represent as uniform data representation is required. Mapping services map the data from WSN to the respective cloud. This service involves entity to entity mapping as well as attribute to attribute mapping that are stored in the mapping repository which contains WSN wise mapping information in a well formatted XML. Mapping strategy is agreed at the time of registration of the WSN. 


\section{DCDS:}

The incoming data coming various sources needs to be treated and formatted before it is stored in the cloud database. As the data could be involving various unwanted, null, missing, irrelevant, multiple, spurious, duplicate data. Hence, the data that we receive has to be cleansed before it in interpreted.

\section{DWS:}

This serviced mainly a stores of data based on various dimensions and facts in different forms depending upton the domain subjects. Various components of data warehouse like, ETL, data load, data refreshment, data storage in various forms of aggregates and finally data retrieval using various technologies like Data mining, Reports, OLAP(Online Analytical Processing), Reports using power BI, etc. It basically contains domain specific enterprise wide information covering every part of the enterprise. Hence the design of the data warehouse differs from that of RDBMS (Relational database system) which is called as MDDB(Multi dimensional database). The design of MDDB uses Start schema and snow flake schema similar to as RDBMS uses E-R diagram (Entity Relationship Diagram).

\section{E. DMS:}

The mining service helps to extract patterns and association on the data. This information can be used in making decisions. Data mining may be performed without data warehouse too, but the presence of data warehouse unit, would drastically help in performance of the system as the data is already cleansed in the ETL component of the warehouse and the data extracted in the warehouse was domain specific.

\section{F. ENS:}

The subscriber repository stores the predicted for the event of interest that at the time of registration and it runs in a continuous manner for the event of the interest that is predicted at the time

\section{G. DVS:}

The large volume of incoming data along with its complexities of data retrieval from subscriber's query pushes the cloud to go for virtual databases where the task is handled bt number of remote hosts clustered together. Where it becomes possible for using the host of WSN itself and visualizes the cloud database on them. The service will take part in allocation of data and query in real time. In this way a huge cost cutting on the cloud infrastructure can be made possible.

\section{H. DMS:}

The maintenance part that is need is taken care by the DMS service. It takes care of the huge data that is generated by the sensor, monitors the good condition and triggers in case of any unusual condition and corrective actions too.

All the data are pulled in gathered data through web service. The data collected in year wise data of sensor network parameters, data storage year wise individually, registry data, parameter master tables.

Traditionally analysts have performed the task of extracting useful information from recorded data. But the increasing volume of data in modern business and science calls for computer-based approaches. As data sets have grown in size and complexity, there has been an unavoidable shift away from direct hands-on data analysis toward indirect, automatic data analysis using more complex and sophisticated tools.

The sensor data that is being sent can be stored in Multi Dimensional database (MDDB) by using star schema as data designing diagram as shown in the figure (Fig 4 ).This is basically a series of organized tables called dimensions[13]. The main problem with dealing with data is to handle steam data. These data that are available in multilevel intrinsically have to follow the mode long like OLAP, that are beyond the traditional way that is used for traditional RDBMS System database modeling[14].

Nowadays the modern technology has made data collection as an almost effortless task. However, the captured data needs to be converted into information and knowledge to become useful.

We hence develop a relational online analytical processing (ROLAP) with following design

ROLAP are servers are highly scalable.

ROLAP tools evaluate huge volumes of data across multiple dimensions.

ROLAP tools store and analyze extremely unstable and unreliable data.

Following are is the star schema -

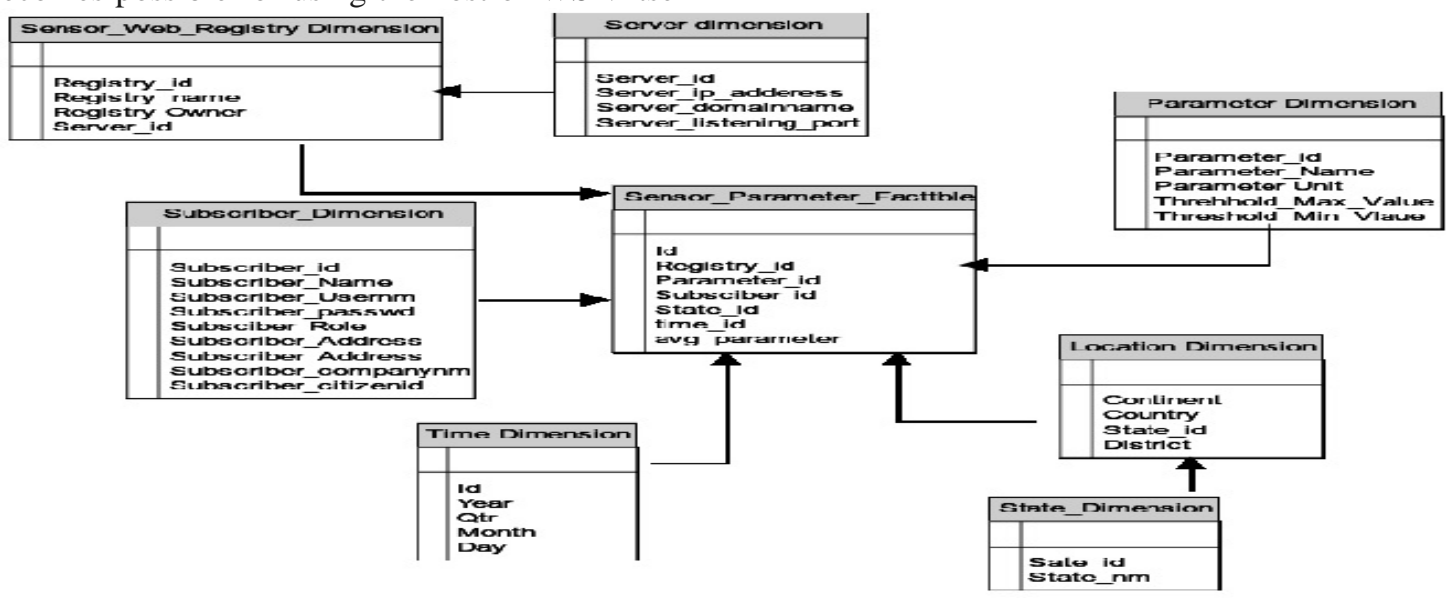

Fig. 4 Star Schema 
Sensor web registry dimension, parameter dimension, Sensor dimension, subscriber dimension, time dimension, location dimension and a sensor_parameter_registry_fact table. The figure blow shows the snowfall schema with aggregate factable. Select ([measures].subscriber_id, [measures].avg_parameter)

on COLUMNS,

(

[server_dimension]

) on ROWS

From Server

Once the star schema is created, as start schema is built over RDBMS database, we then generate a cube known as ROLAP cube. On which we can then perform various operation such as Roll-up,Drill-down,Slice and dice,Pivot (rotate).

We then generate results using MDX queries (Multidimensional query) as

This star schema then can be accessed later to generate various report in SSRS, Power BI or Tableau.

\section{Data mining model:}

As we see we have sensor data, server dimension, subscriber details, time dimension and data for location dimension.

The first and foremost thing to be done is to understand the data and the distribution form of data. There are of forms as discrete distribution, continuous distribution.

Here we have continuous data hence the distribution is continues distribution that follows the formula:

The probability density function of the distribution continuous uniform is usually converted into discrete or range data by using technique like binning along with cut function to break the data into groups.

The data is then normalized using the min-max approach then apply logistic regression found in sklearn library in python. A binary logistic regression describes the relationship between the dependent binary variable and one or more independent variable/s.

The binary dependent variable has two possible outcomes are:

' 1 ' for true/success; or

' 0 ' for false/failure

By stating the classifier as

classifier

$=$

Logistic

Regression(solver='lbfgs',random_state=0) , we then train the classifier with the following function and test the system created using:

LogisticRegression $(\mathrm{C}=1.0$, class_weight $=$ None, dual $=$ False, fit_intercept=True, intercept_scaling=1, max_iter=100, multi_class='warn', n_jobs=None, penalty $=$ 'l2', random_state $=0$, solver $=$ 'lbfgs', tol $=0.0001$, verbose $=0$, warm_start $=$ False)) with ' 12 ' being the loss function to test the algorithm performance.

Regression algorithm is available in many variants linear approach model to model the relationship between a scalar variable (or dependent variable) and one or more scalar variable, and for more than one explanatory variable, the process is called multiple linear regression. This term is distinct from multivariate linear regression, where multiple correlated dependent variables are predicted, rather than a single scalar variable.

First we use pandas library of python to read the csv files. We make use of pandas - used to create the Data Frame to capture the dataset in Python
We take humidity and temperature (Variable $\mathrm{X}$ ) as input and pressure (variable Y) as target variable.

The data spread with average value of the "pressure" column by using seaborn and matplotlib.

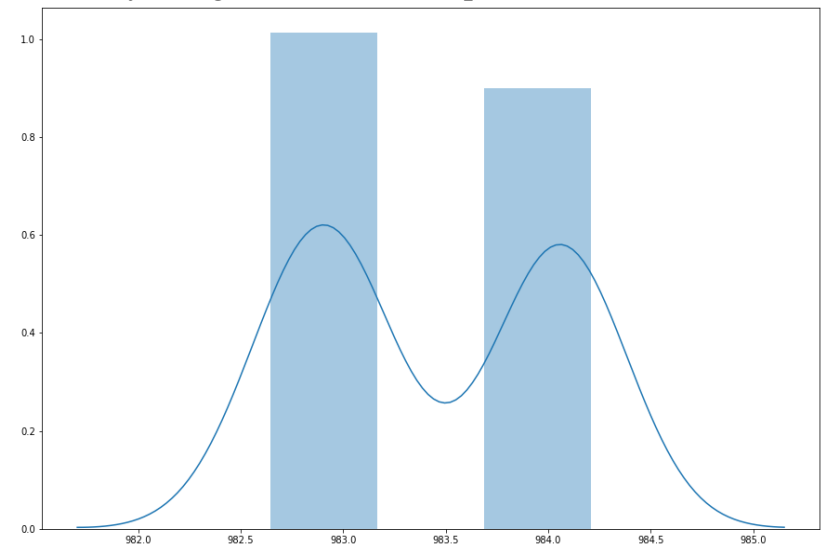

Fig. 5 Seaborn

We then execute the regression algorithm by using sklearnused to build the logistic regression model in Python.

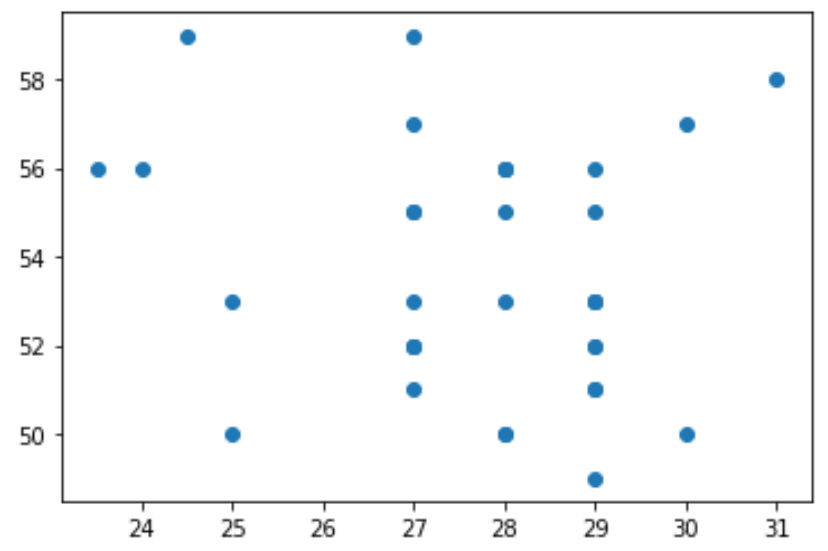

Fig. 6 Scatter plot of humidity and temperature

This above figure shows the scatter plot of humidity and temperature.

We divide the dataset in 80:20 ratio. Where the $80 \%$ of data is taken as training set and $20 \%$ is taken for testing.

Following shows the sample working data output:

Describing data [[ 27. 2885. ] [ 28. 2953. ] [ 28. 2953. ] [ 27. 2885. ] [ 30. 2953. ] [ 25. 2885. ] [ 29. 2885. ] [ 31. 2953. ] [ 25. 2885. ] [ 29. 2885. ] [ 24.5 2953. ] [ 28. 2953. ] [ 27. 2885. ] [ 30. 2953. ] [ 24. 2953. ] [ 29. 2885. ] [ 23.5 2953. ] [ 27. 2898. ] [ 29. 2893. ] [ 27. 2947. ] [ 27. 2869. ] [ 28. 2955. ] [ 28. 2926. ] [ 29. 2922. ] [ 27. 2903. ] [ 28. 2911. ] [ 27. 2886. ] [ 29. 2888. ] [ 29. 2888. ] [ 28. 2895. ] [ 29. 2872. ] [ 29. 2931. ] [ 27. 2932. ] [ 29. 2857. ]]

As we have multiple variables we go for multiple linear regression. Hence the coefficient variables has to be determined.

coefficient of determination: 0.3084992047533225 , where in the coefficient of determination is denoted as $\mathrm{R}^{2}$ or $\mathrm{r}^{2}$ and pronounced "R squared", is the proportion of the variance in the dependent variable that is predictable from the independent.

It is one of the loss functions that 
determine the accuracy of the model. This tells us a numerical value obtained by squaring Pearson's correlation coefficient. It is the percentage of the variance of the dependent variable that could account by relationship with the independent variable.

intercept: [952.51890621]

intercept is simply the expected mean value of $\mathrm{Y}$ at that value

slope: [[0.03268717 0.01030675]]

Slope is calculated by finding $\mathrm{b}$ as the covariance of $\mathrm{x}$ and $\mathrm{y}$, divided by the sum of squares (variance) of $x$.

Prediction done with the test data is as follows:

Predicted response: [[983.13644404] [983.86999046]

[983.86999046] [983.13644404] [983.93536481]

[983.07106969] [983.20181839]

[983.96805198]

[983.07106969]

[983.20181839]

[983.75558535]

[983.86999046]

[983.73924176]

[983.13644404]

[983.93536481]

[983.27043184]

[983.20181839]

[983.72289818]

[983.28427242]

[983.77546276]

[982.97153598]

[983.58316827]

[983.89060397]

[983.59170811]

[983.1467508]

[983.32196561]

[983.23273865]

[983.43710681]

[983.27219875]

[983.06783059]

[983.23273865]

[983.67592906]

[983.62086146] [982.91322929]]

The OLAP developed for the real streaming data from the sensor networks is calibrated and tested a machine learning model which finds a relationship between the temperature and humidity with the atmospheric pressure.

\section{CONCLUSION}

A complete architecture of cloud based Sensor Network is proposed and experimetal work has been done in the data service layer. Sensor Networks are known to have the continuous streaming data and it is demonstrated how this data can be captured in real time and can be processed with the help of existing data analytics application like tensorflow and python. This is just a sample as how things can be done. All diverse networks can be integrated in this way and the data captured can be disseminated, universalized and cleaned for the purpose of its interpretation and real time use. Dong this can facilitate a global understanding of the data and enable us to tackle various real world problems like pollution and global warming.

\section{REFERENCES}

1. Kobialka T, Buyya R, Leckie C, Kotagiri R (2007) A sensor web middleware with stateful services for heterogeneous sensor networks. In: Proceedings 3rd international conference on intelligent sensors, sensor networks and information processing (ISSNIP 2007). Melbourne, Australia, December 2007 pp 491-496

2. Mohamed, Nader, and Jameela Al-Jaroodi. "A survey on serviceoriented middleware for wireless sensor networks." Service Oriented Computing and Applications 5.2 (2011): 71-85.

3. Levent G, Claudia R, Cyril L, Andre B, and VincentO (2008) SstreaMWare: A service oriented middleware for heterogeneous sensor data management. In: Proceedings of ICPS, Sorrento, Italy, July 2008 pp 121-130

4. Chu X, Kobialka T, Durnota B, Buyya R (2006) Open sensor web architecture: core services. In: Proceedings of 4th international conference on intelligent sensing and information processing. Bangalore, India, December 2006 p. 98-103

5. Dunstan N. (2013) An OLAP Server for Sensor Networks Using Augmented Statistics Trees. In: Li J.etal. (eds) Trends and Applications in Knowledge Discovery and Data Mining. PAKDD 2013. Lecture Notes in Computer Science, vol 7867. Springer, Berlin, Heidelberg.
6. Cuzzocrea, Alfredo, Filippo Furfaro, Elio Masciari and Domenico Saccà. "Improving OLAP Analysis of Multidimensional Data Streams via Efficient Compression Techniques." Intelligent Techniques for Warehousing and Mining Sensor Network Data. IGI Global, 2010. 17-49. Web. 17 Apr. 2020. doi:10.4018/978-1-60566-328-9.ch002

7. -REPORT-2006-006, Ecole Polytechnique F'ed'erale de Lausanne, 2006

8. .url; http://lsirpeople.epfl.ch/hauswirth/papers/LSIR-REPORT-2006006.pdf Botts, Mike, et al. "OGC® sensor web enablement: Overview and high level architecture." GeoSensor networks. Springer Berlin Heidelberg, 2008. 175-190.

9. Mike Botts, George Percivall, Carl Reed, John Davidson., (2006), "OGC Sensor Web Enablement: Overview and High Level Architecture", Open Geospatial Consortium, OGC White Paper, OGC 06-050r2 v2, 19th July 2006

10. Kamlendu Pandey, S.V. Patel ,Design of SOA based Sensor Web Registry Proceedings of CICSyn IEEE confrence, Indore July,2009 P.

11. Kamlendu Pandey, S.V. Patel ,Design of SOA based Service Stack for Collaborative Wireless Sensor Network Submitted in WSCN 2009, IEEE Conference, Allahabad

12. Amundson I, Kushwaha M, Koutsoukos X, Neema S, Sztipanovits J (2006) OASiS: a service-oriented middleware for pervasive ambientaware sensor networks. In: Pervasive and mobile computing journal on middleware for pervasive computing, October 2006 URL: http://folk.uio.no/paalee/referencing_publications/ref-wsnamundson_report2006.pdf

13. K. Aberer, Ali Sulehi.,'The Global Sensor Networks, middleware for efficient and flexible deployment and interconnection of sensor networks", Tech. Rep. LSIR

\section{AUTHORS PROFILE}

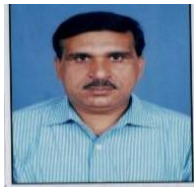

Dr. Kamlendu Kumar Pandey is Assistant Professor in Dept. Of Information and Communication Technology at Veer Narmad South Gujarat University, Surat, Gujarat in India. The interest areas are Wireless Sensor Networks, Cloud Computing, and Service Oriented Architecture. He is having 18 years of rich experience in academics and industry in the various fields of Computer Science.

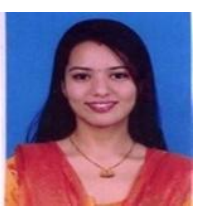

Dr. Veena Jokhakar received B.Sc.(Information Technology), M.Sc.(Information Technology) and Ph.d. (Computer Science) Degree from Veer Narmad South Gujarat University in the years 2004, 2006 and 2017 respectively. She has industry and academic experience of 13 years. Her area of interests and research area are viz. Data Mining, Data Science and Data Warehouse. 